\title{
Microbiologia de sistema de lodos ativados e sua relação com o tratamento de efluentes industriais: a experiência da Cetrel
}

\author{
Activated sludge system microbiology and its relationship with the industrial \\ effluents treatment: the experience of the Cetrel
}

\author{
Gisele Santos Silva de Oliveira \\ Bióloga pela Universidade Católica do Salvador (UCSal). Pesquisadora da Central de Tratamento de Efluentes Líquidos (Cetrel) Proteção Ambiental \\ Cristiano Venícius de Matos Araújo \\ Biólogo pela UCSal. Mestre em Ecologia e Biomonitoramento pela Universidade Federal da Bahia (UFBA). Doutorando pela Universidade de Cádiz, \\ Espanha (CSIC)
}

\section{José Gilson Santos Fernandes}

Químico Industrial pela Universidade Estadual da Paraíba (UEPB). Mestre em Engenharia Civil pela Universidade Estadual da Paraíba (UFPB). Engenheiro da Cetrel Proteção Ambiental

\begin{abstract}
Resumo
Buscou-se, neste trabalho, caracterizar a comunidade microbiana de lodos ativados dos tanques de aeração (TA-2, TA-3 e TA-4) da estação de tratamento de efluentes (ETE) da Cetrel e correlacioná-la com parâmetros físico-químicos de processo, bem como avaliar o impacto do efluente industrial (El) bruto na estrutura da comunidade do lodo. Adicionalmente, foi verificada a existência de possíveis correlações entre a qualidade do efluente tratado final (ETF) e a comunidade do lodo para identificar algum grupo biológico que pudesse ser usado como bioindicador desta ETE. Os principais grupos encontrados nos TA foram ciliados (livre natantes, de vida livre e pedunculados) e flagelados. Verificou-se que altas cargas de DBO e DQO no El afetam negativamente o sistema, especialmente o grupo dos ciliados, que foram os melhores indicadores da elevada eficiência da ETE. O TA-2 mostrou ser o tanque mais estável e eficiente.
\end{abstract}

Palavras-chave: biodegradação; estação de tratamento de efluentes; Pólo Industrial de Camaçari; protozoários.

\begin{abstract}
The purpose of this study was to characterize the microbial community of activated sludge in the aeration tanks (AT-2, AT-3 and AT-4) from the Cetrel wastewater treatment plant (WWTP) and to correlate it to physical-chemical parameters, as well as to assess the impact of the raw industrial effluent (IE) on the activated sludge community. Additionally, it was verified the relationship between the treated effluent (TE) quality and the biological groups to determinate indicators of the WWTP efficiency. Ciliated protozoa (free-swimming, crawler and sessile) and flagellates were the main biological groups found in the TA. It was demonstrated that high IE-BOD and -COD loads have a negative influence on the system, especially on the ciliated protozoa, which was the best indicator of high efficiency of the WWTP. The AT-2 was the more stable and efficient.
\end{abstract}

Keywords: biodegradation; wastewater treatment plant; Industrial Complex of Camaçari; protozoa.

\section{Introdução}

Os resíduos industriais e domésticos decorrentes das atividades humanas devem ser adequadamente tratados em estação de tratamento de efluentes (ETE), a fim de remover a maior quantidade possível de poluentes, minimizar os efeitos adversos ao ambiente, prevenir a poluição e proteger a saúde pública (LAMBOLEZ et al,
1994; FERNÁNDEZ et al, 1995). Um tratamento de efluente adequado exige rigoroso controle do sistema utilizado, entendimento sobre a influência dos compostos tóxicos no processo de depuração e quão eficiente é o sistema para remoção da carga tóxica, a qual, muitas vezes, é medida pela redução de DQO (demanda química de oxigênio), DBO (demanda bioquímica de oxigênio), toxicidade, ou outro composto cuja remoção é indispensável 
para disposição final (PATOINE et al, 1997; UBAY ÇOKGÖR et al, 1998; ARAÚjO et al, 2005; BEAL et al, 2006; SANTOS et al, 2006). Os tratamentos baseados em processos biológicos permitem tratar grandes volumes de efluente, apresentam menor custo de funcionamento e simplicidade operacional (FREIRE et al, 2000; DA MOTTA et al, 2003), sendo que para efluentes complexos, o processo biológico mais amplamente usado é o tratamento por lodos ativados, cujo nível de eficiência é elevado, embora o processo anaeróbico tenha mostrado bom desempenho (JENKINS; RICHARD; DAIGGER, 2003).

O sistema de lodos ativados consiste em uma complexa associação de micro-organismos composta por bactérias, protozoários, fungos e micrometazoários que oxidam os compostos orgânicos e inorgânicos presentes nos efluentes (BENTO et al, 2005; DAVIES, 2005). A comunidade estabelecida nesse sistema é dinâmica e fundamental ao tratamento, sendo que cada espécie tem sua importância para o bom funcionamento do sistema (AMMAN; GLOCKNER; NEEF, 1997). A estrutura dessa comunidade apresenta forte relação com as condições operacionais e com a qualidade e quantidade de efluente que alimenta o processo (VAZOLLÉR et al, 1989), de modo que a avaliação microbiológica do lodo é capaz de fornecer informações sobre o desempenho da ETE (POOLE, 1984). Mudanças nas condições ambientais provenientes da composição do afluente do reator, ou mesmo da operação da estação, podem levar a alterações na comunidade do lodo, influenciando os processos de biodegradação e, portanto, reduzindo a qualidade do efluente tratado (MADONI et al, 1996; FORNEY et al, 2001; DALZELL et al, 2002). Embora a caracterização microscópica da comunidade do lodo ativado possua grande importância para a avaliação das condições das ETE, o uso de tal ferramenta ainda é incipiente no Brasil, e os resultados são, em geral, subutilizados (BENTO et al, 2005).

A ETE da Central de Tratamento de Efluentes Líquidos (Cetrel) trata os efluentes provenientes das indústrias do Pólo Industrial de Camaçari (PIC), Bahia, Brasil, usando o sistema de lodos ativados, cuja comunidade é exposta às mais variadas substâncias tóxicas do efluente industrial (EI), o que pode levar a uma alteração nessa microbiota e a perdas na eficiência do tratamento. Para o monitoramento da ETE, foram analisados alguns parâmetros físico-químicos no EI, nos tanques de aeração (TA) e no efluente tratado final (ETF). Entretanto, faz-se necessária uma caracterização da comunidade do lodo ativado e uma avaliação de seu comportamento em função das características do EI e dos controles operacionais adotados nos TA. Assim, este estudo tem como objetivo caracterizar a comunidade microbiana do lodo ativado da ETE da Cetrel, identificando os principais grupos de micro-organismos (protozoários e micrometazoários) encontrados nos TA, e correlacionar a frequência desses grupos com parâmetros físico-químicos e toxicológicos do EI para avaliar como a comunidade pode ser afetada pelo contato com o efluente bruto. Buscou-se, também, correlacionar essa comunidade aos controles operacionais, visando a identificar os efeitos dos parâmetros de processo sobre sua estrutura. Adicionalmente, verificouse a existência de possíveis correlações entre a qualidade do ETF e os organismos presentes no processo de tratamento na busca de identificar algum grupo que pudesse ser usado como bioindicador da qualidade do efluente tratado e da eficiência da ETE. Com base nessa caracterização, avaliou-se o desempenho dos TA que compõem a ETE da Cetrel.

\section{Material e métodos}

\section{Área de estudo}

Este estudo foi realizado na ETE da Cetrel Proteção Ambiental, localizada na área do PIC (maior complexo industrial integrado da América Latina), que atualmente trata os efluentes de mais de 50 indústrias ali instaladas. A capacidade operacional dessa ETE é de $167.232 \mathrm{~m}^{3}$ de efluente por dia e uma carga máxima de DQO de $330.663 \mathrm{~kg} / \mathrm{dia}$ (CETREL, 2004). As etapas que compõem o sistema de tratamento de efluentes da Cetrel estão simplificadas no esquema baseado em Araújo et al (2005) (Figura 1). Após todo o processo de tratamento, o ETF é conduzido para o mar através do emissário submarino. 


\section{Tanques de aeração}

A ETE da Cetrel possui, atualmente, três TA (TA-2, TA-3 e TA-4) que operam simultaneamente, sem interligação. O TA-2 tem capacidade para $39.600 \mathrm{~m}^{3}$, sendo constituído por dez aeradores de 175 CV cada e com capacidade para uma vazão máxima de 1.485 $\mathrm{m}^{3} / \mathrm{h}, 67.417 \mathrm{~kg} /$ dia de carga de DQO e $24.969 \mathrm{~kg} /$ dia de carga de $\mathrm{DBO}$, com mistura do tipo carrossel. O efluente gerado nesse TA após tratamento é chamado ETF-1. O TA-3 opera com um sistema de mistura completa (convencional), tem capacidade para 48.000 $\mathrm{m}^{3}$, constituído por 20 aeradores de $150 \mathrm{CV}$ cada, com uma vazão de $2.000 \mathrm{~m}^{3} / \mathrm{h}$, carga de DQO de $115.572 \mathrm{~kg} /$ dia e carga de DBO de $42.804 \mathrm{~kg} / \mathrm{dia}$, sendo que o efluente gerado, misturado ao proveniente do TA-4, compõe o ETF-2. O TA-4 tem capacidade para $50.000 \mathrm{~m}^{3}$, com 14 aeradores de $150 \mathrm{CV}$ cada e oito misturadores submersíveis de $6,2 \mathrm{CV}$ cada. A vazão máxima é de $2.167 \mathrm{~m}^{3} / \mathrm{h}$, carga de DQO de $89.889 \mathrm{~kg} /$ dia e $33.292 \mathrm{~kg} /$ dia de DBO, sendo a mistura similar à do TA-2, tipo carrossel. O efluente gerado nesse TA, juntamente com o do TA-3, compõe o ETF-2. O efluente tratado final (ETF) é uma mescla do ETF-1 (gerado no TA-2) com o ETF-2 (gerado nos TA-3 e TA-4).

\section{Amostragem}

A amostragem do lodo dos três TA foi realizada em frascos de vidro tipo âmbar, não preenchidos completamente, a fim de se manter o oxigênio suficiente para os organismos até o momento da avaliação microbiológica, que ocorreu logo após a coleta. As amostras do EI foram coletadas em frascos de polietileno, antes da bacia de equalização (BEQ) (Figura 1), e as do ETF, após os decantadores secundários (Figura 1). As denominações efluente industrial (EI) e efluente tratado final (ETF) foram mantidas, pois tais termos já estão caracterizados na Cetrel, embora o EI represente um afluente para a ETE. O período de amostragem do lodo e dos efluentes compreendeu os meses de maio a novembro de 2005 e de fevereiro a maio de 2006 ( $\mathrm{n}=75)$.

\section{Avaliação biológica}

$\mathrm{Na}$ avaliação biológica da comunidade do lodo, buscou-se identificar, através de análises microscópicas, os ciliados de vida livre (CVL), ciliados livre natantes (CLN), ciliados pedunculados (CP), suctórias, flagelados, amebas, nematoides e rotíferos. A identificação dos organismos foi baseada em Kudo (1931), Westphal (1977), Needham e Needham (1978), realizada até nível de gênero. A avaliação consistiu na retirada de uma alíquota de $60 \mu \mathrm{L}$ do sobrenadante do licor misto (mistura efluente e lodo), juntamente com $20 \mu \mathrm{L}$ do lodo sedimentado, no qual se concentrava a maior parte dos organismos, para montagem de lâmina e posterior observação de todos os campos no microscópico óptico. A cada análise, os grupos foram classificados em ausentes, raros, frequentes e abundantes (Tabela 1). Todas as análises foram realizadas em duplicata. A partir dessa análise, pôde-se relacionar a presença de organismos de determinados grupos taxonômicos com o funcionamento da ETE. A frequência de observação para classificação dos organismos é uma escala arbitrária usada como parâmetro de controle nessa ETE, visando a acompanhar e comparar o desempenho dos TA.

\section{Parâmetros avaliados}

As variáveis determinadas dentro dos tanques foram sólidos suspensos (SS), sólidos sedimentáveis (SSD) (APHA/AWWA/WEF, 2005) e oxigênio dissolvido (OD) (sensor Mettler Toledo). A caracterização do EI baseou-se na $\mathrm{DBO}_{5}, \mathrm{DQO}, \mathrm{pH}, \mathrm{N}-\mathrm{NH}_{3}$, carbono orgânico total (COT) (APHA/AWWA/WEF, 2005), taxa de consumo de oxigênio (TCO) (FERNANDES et al, 2001) e toxicidade pelo sistema-teste Microtox (MICROBICS CORPORATION, 1998). Nas amostras de ETF-1 (provenientes do TA-2) e ETF-2 (provenientes dos TA-3 e TA-4) foram avaliadas as concentrações de SS e DQO (APHA/AWWA/WEF, 2005). Realizaram-se análises de DQO, DBO e toxicidade do ETF, para posterior cálculo da redução dessas variáveis em relação ao efluente de entrada. Para comparação entre as amostras de efluentes e lodo, obedeceu-se o tempo de retenção hidráulica na estação desde a entrada do EI até sua saída como ETF (ver Figura 1). As amostras do EI são referentes ao dia anterior à análise do lodo, para obedecer ao tempo de residência de \pm 24 h na BEQ. Por outro lado, os dados dos ETF-1 e ETF-2 são relativos ao dia posterior à caracterização do lodo, devido ao tempo de retenção de \pm 24 h do efluente desde a saída do TA, passagem pelo decantador e posterior coleta. Todas as análises para caracterização dessas amostras foram realizadas no mesmo dia da coleta.

\section{Análises estatísticas}

As correlações entre as variáveis biológicas (grupos) e variáveis físico-químicas e de toxicidade, tanto do licor misto, quanto do EI, ETF-1 e ETF-2, foram verificadas pelo método de correlação

Tabela 1 - Valores atribuídos à frequência dos organismos encontrados nos TA

\begin{tabular}{lcc|}
\hline Classificação & Valores & Frequência de observação \\
\hline Ausente & 0 & Não observados \\
\hline Raro & 1 & 1 a 15 organismos \\
\hline Frequente & 2 & 16 a 30 organismos \\
\hline Abundante & 3 & Acima de 30 organismos
\end{tabular}


não-paramétrica de Spearman (ZAR, 1996), utilizando o programa GraphPad Instat versão 3.0. As correlações (positivas, indicando relação direta, e negativas quando estavam inversamente relacionadas) foram consideradas significativas com $p<0,05$. Para as análises de correlação, a medida de toxicidade dada em $\mathrm{CE}_{50}$ (concentração que causa redução de luminescência da bactéria em $50 \%$ ), por ser inversamente proporcional ao efeito tóxico, foram transformadas em unidades de toxicidade $\left(100 / \mathrm{CE}_{50}\right)$, tornando o valor diretamente proporcional à toxicidade (MICROBICS CORPORATION, 1998). Os cálculos para remoção de DBO, DQO e toxicidade (ARAÚJO et al, 2005) foram realizados nas amostras compostas do ETF, formadas pelo ETF-1 e ETF-2, usando a seguinte equação:

$R(v a r)=\left[1-\left(\frac{v a r-E T F}{v a r-E I}\right)\right] \times 100$

Equação 1

onde:

$R(v a r)$ : valor em percentagem da redução da variável a ser medida (DQO, DBO, ou toxicidade);

var-ETF: valor da variável medida nas amostras de ETF; var-EI: valor da variável medida nas amostras de EI.

Tabela 2 - Organismos encontrados no lodo ativado e sua ocorrência dentro dos TA

\begin{tabular}{|c|c|c|c|}
\hline Classe & Grupo & Gênero & Ocorrência \\
\hline \multirow[t]{9}{*}{ Ciliata } & $\begin{array}{l}\text { Ciliados de vida } \\
\text { livre (CVL) }\end{array}$ & Aspidisca & TA-2 e TA-3 \\
\hline & \multirow[t]{3}{*}{$\begin{array}{l}\text { Ciliados livre } \\
\text { natantes (CLN) }\end{array}$} & $\begin{array}{l}\text { Chilodonella } \\
\text { Loxodes } \\
\text { Paramecium } \\
\text { Spirostomum }\end{array}$ & $\begin{array}{l}\text { TA-2, TA-3 e TA-4 } \\
\text { TA-2 e TA-3 } \\
\text { TA-2 } \\
\text { TA-2 e TA-3 }\end{array}$ \\
\hline & & Stylonychia & TA-2 \\
\hline & & Uronema & TA-2, TA-3 e TA-4 \\
\hline & \multirow{3}{*}{$\begin{array}{l}\text { Ciliados } \\
\text { pedunculados } \\
(\mathrm{CP})\end{array}$} & Epistylis & TA-2, TA-3 e TA-4 \\
\hline & & Opercularia & TA-2, TA-3 e TA-4 \\
\hline & & Vorticella & TA-2, TA-3 e TA-4 \\
\hline & \multirow[t]{2}{*}{ Suctória } & Podophrya & TA-2 \\
\hline & & Tokophrya & TA-2 \\
\hline \multirow[t]{4}{*}{ Flagellata } & \multirow[t]{4}{*}{ Flagelados } & Bodo & TA-2, TA-3 e TA-4 \\
\hline & & Heteronema & TA-2, TA-3 e TA-4 \\
\hline & & Monas & TA-2, TA-3 e TA-4 \\
\hline & & Paranema & TA-2, TA-3 e TA-4 \\
\hline \multirow[t]{3}{*}{ Sarcodina } & \multirow[t]{3}{*}{ Amebas } & Amoeba & TA-2 \\
\hline & & Arcella & TA-2 \\
\hline & & Difflugia & TA-2 \\
\hline Nematoda & Nematoides & Rhabditis & TA-2 \\
\hline
\end{tabular}

\section{Resultados e discussão}

Os resultados da caracterização microbiológica do lodo mostraram que os flagelados, CP e CLN foram os grupos mais frequentes, encontrados nos três TA (Tabela 2). Dos grupos analisados, apenas os rotíferos não foram encontrados nos TA. O TA-2 apresentou todos os grupos de organismos identificados e sempre com maior frequência do que nos demais tanques. Suctórias, nematoides e amebas somente foram encontrados no TA-2, sendo que os organismos pertencentes aos dois primeiros grupos apenas foram observados em duas amostragens, por isso não foram utilizados para comparação. Conforme Costa et al (2003), abundância e biodiversidade elevadas sugerem um ambiente com menor grau de estresse e maior estabilidade, o que foi observado no TA-2 e, portanto, o caracterizou como o mais estável. A análise do TA-4 mostrou ser esse o menos diverso, contendo apenas CLN, CP e flagelados, o último grupo o mais frequente. Os protozoários e micrometazoários fornecem informações úteis sobre a atividade biológica do lodo, podendo avaliar a qualidade do efluente e o desempenho da estação, além de atuarem como polidores dos efluentes (MADONI, 1994; VAZOLLÉR, 1999). Análises rotineiras da microbiota como indicadoras do desempenho de sistemas têm sido amplamente descritas (VAZOLLÉR, 1999; BENTO et al, 2005). Madoni (1994) mostrou que o número de espécies morfológicas reduzido é indicativo de menor eficiência no tratamento em comparação a um sistema mais diverso. Embora o TA-3 não tenha apresentado diversidade similar à observada no TA-2, foi mais diverso do que o TA-4, sugerindo que o TA-2 foi mais eficiente, seguido do TA-3 e TA-4.

As análises de correlação foram realizadas com os grupos cuja ocorrência foi significativa ao longo do estudo. Desse modo, no TA-2 foram utilizados os dados dos CLN, CP, CVL, flagelados e amebas, e para os TA-3 e TA-4 utilizaram-se os dados dos grupos CP, CLN e flagelados. Os valores de DBO e DQO do EI apresentaram correlação negativa (aumento da DQO e da DBO implica em redução da biodiversidade nos TA) com todos os grupos de organismos (Tabela 3), o que corrobora com os dados encontrados em outros estudos (POOLE, 1984; SALVADÓ; GRACIA, 1993; SALVADÓ; GRACIA; AMIGÓ, 1995; BENTO et al, 2005). Os CLN e CVL, no TA-2, parecem ser mais afetados com cargas elevadas de DBO. Tal sensibilidade foi verificada por Salvadó e Gracia (1993), quando registraram o decréscimo da diversidade de ciliados quando do aumento da carga orgânica do efluente. O mesmo foi documentado por Costa et al (2003), que observaram a diminuição no número de ciliados e parada na movimentação dos cílios nessas mesmas condições. No TA-3, a mesma tendência foi verificada para os CP, com correlação de -0,42, e no TA-4 a correlação foi mais evidente com os CP e flagelados (-0,35) (Tabela 3). Quanto à carga de DQO, os CVL e flagelados correlacionaram-se negativamente no TA-2. No TA-3 
Tabela 3 - Coeficientes de correlação entre os grupos biológicos dos TA-2, TA-3 e TA-4 e os parâmetros DBO e DQO do El

\begin{tabular}{|c|c|c|c|c|c|c|}
\hline \multirow{2}{*}{ Grupos } & \multicolumn{3}{|c|}{ DBO } & \multicolumn{3}{|c|}{ DQO } \\
\hline & TA-2 & TA-3 & TA-4 & TA-2 & TA-3 & TA-4 \\
\hline Ciliados livre natantes & $-0,23^{*}$ & $-0,20$ & $-0,14$ & $-0,20$ & $-0,01$ & 0,03 \\
\hline Ciliados de vida livre & $-0,45^{\star}$ & - & - & $-0,34^{*}$ & - & - \\
\hline Ciliados pedunculados & $-0,22$ & $-0,42^{*}$ & $-0,35^{\star}$ & $-0,20$ & $-0,26^{\star}$ & $0,23^{*}$ \\
\hline Amebas & $-0,18$ & - & - & $-0,12$ & - & - \\
\hline Flagelados & $-0,12$ & $-0,20$ & $-0,35^{*}$ & $-0,25^{*}$ & $-0,22$ & $0,31^{*}$ \\
\hline
\end{tabular}

* $p<0,05$.

tal correlação foi observada com os CP $(-0,26)$, o mesmo ocorrendo no TA-4, no qual também foi registrada correlação negativa entre os valores de DQO e flagelados (Tabela 3). Essas correlações negativas podem ser avaliadas como indicativo da sensibilidade dos organismos a elevadas cargas de DBO e DQO do EI. No TA-3, a presença de $\mathrm{CP}$ indicou cargas mais baixas de DBO e DQO, enquanto no TA-4 a melhor referência para DBO e DQO baixas seria a abundância dos $\mathrm{CP}$ e flagelados, e não necessariamente suas ocorrências, pois as mudanças na estrutura da comunidade desse tanque se resumiram à frequência dos organismos e não à diversidade, uma vez que esses grupos sempre foram encontrados nesse TA. Os CVL no TA-2 foram os melhores indicadores de um efluente de entrada com cargas de DBO e DQO baixas.

A única correlação com o pH do EI ocorreu com os $\mathrm{CP}(0,30)$ no TA-4 (Tabela 4). Durante todo o período estudado, os valores de $\mathrm{pH}$ do EI variaram entre 7,2 e 8,9. Em geral, o sistema de lodos ativados deve operar numa faixa de pH entre 6,5 a 8,5 (VON SPERLING, 1997). A relação entre alguns parâmetros físico-químicos, a exemplo do pH e uma determinada espécie, não segue necessariamente um padrão linear, pois há uma faixa ótima de desenvolvimento, sendo que acima ou abaixo dela a espécie pode diminuir sua densidade ou mesmo desaparecer (SALVADÓ; GRACIA; AMIGÓ, 1995). A correlação encontrada com os CP pode estar relacionada não apenas ao pH, mas também à associação desse parâmetro com as condições de processo estabelecidas no tanque em questão.

Os CVL, no TA-2, apresentaram correlação negativa com a concentração do N-NH ${ }_{3}$ do EI $(-0,26)$ (Tabela 4). Vazollér et al (1989) e Bento et al (2005) relataram a ocorrência de nitrificação associada à presença de Aspidisca (CVL), demonstrando que a ocorrência dessa espécie é beneficiada com a redução de N-NH . As amebas também são conhecidas pela sensibilidade à amônia, indicando ocorrência de nitrificação no tanque (MADONI; DAVOLI; CHIERICH, 1993), porém tal comportamento não foi aqui atestado. De acordo com Madoni, Davoli e Chierich (1993), em um mesmo grupo, algumas espécies podem ser sensíveis à uma variável, enquanto outras podem ser mais resistentes. Varginicola crystallina (CP) refletiu a ocorrência de nitrificação, enquanto Vorticella microstoma e Opercularia
Tabela 4 - Coeficientes de correlação entre os grupos biológicos dos TA-2, TA-3 e TA-4 e os parâmetros pH e N-NH ${ }_{3}$ do El

\begin{tabular}{lcccccc} 
& \multicolumn{3}{c}{$\mathrm{pH}$} & \multicolumn{3}{c}{$\mathrm{N}^{*} \mathrm{NH}_{3}$} \\
\cline { 2 - 8 } Grupos & $\mathrm{TA}-2$ & $\mathrm{TA}-3$ & $\mathrm{TA}-4$ & $\mathrm{TA}-2$ & $\mathrm{TA}-3$ & $\mathrm{TA}-4$ \\
\hline Ciliados livre natantes & $-0,04$ & 0,08 & 0,05 & $-0,21$ & 0,10 & $-0,18$ \\
\hline Ciliados de vida livre & 0,03 & - & - & $-0,26^{*}$ & - & - \\
\hline Ciliados pedunculados & $-0,01$ & 0,23 & $0,30^{*}$ & 0,02 & $-0,14$ & $-0,14$ \\
\hline Amebas & 0,10 & - & - & 0,16 & - & - \\
\hline Flagelados & 0,20 & 0,11 & 0,16 & 0,06 & $-0,23$ & $-0,20$
\end{tabular}

* $p<0,05$.

spp (ambos CP) mostraram relação inversa com a qualidade do efluente tratado, ocorrendo em baixas concentrações de oxigênio no tanque e com elevadas concentrações de DBO no efluente tratado (MADONI; DAVOLI; CHIERICH, 1993; MADONI, 1994). Os resultados aqui apresentados permitem atestar a sensibilidade dos CVL à alta concentração de N-NH 3 no EI. Nos TA-3 e TA-4 não se observou correlação significativa.

COT e TCO não apresentaram correlação com os organismos encontrados nos TA (dados não mostrados). Como a análise de COT inclui compostos de carbono orgânico estáveis que não são oxidados biologicamente (DAVIES, 2005), torna-se difícil qualquer relação com a microbiota do lodo. A TCO é obtida através das variações das concentrações de OD no licor misto ao longo do tempo, quando não se aplica aeração, sendo utilizada para determinar a velocidade da atividade biológica nos sistemas de tratamento aeróbio com lodo em suspensão (FERNANDES et al, 2001). De acordo com esses autores, uma redução na taxa de consumo de oxigênio, sem diminuição da carga orgânica aplicada, pode ser indício de substâncias tóxicas ou inibidoras no sistema. Embora a carga do EI apresente relativa variação, com oscilações na TCO, em geral, o sistema de lodos ativados da Cetrel, desde que fornecida aeração necessária, alcança elevadas taxas de depuração (OLIVEIRA et al, 2007).

Apenas com os CVL, no TA-2, houve correlação positiva significativa $(0,38)$ com a toxicidade do EI (Tabela 5). Seria provável que 
Tabela 5 - Coeficientes de correlação entre os grupos biológicos dos TA-2, TA-3 e TA-4 e a ecotoxicidade (sistema-teste Microtox) do EI

\begin{tabular}{lccc} 
& \multicolumn{3}{c}{ Toxicidade } \\
\cline { 2 - 4 } Grupos & TA-2 & TA-3 & TA-4 \\
\hline Ciliados livre natantes & 0,15 & 0,07 & 0,06 \\
\hline Ciliados de vida livre & $0,38^{*}$ & - & - \\
\hline Ciliados pedunculados & 0,11 & 0,14 & 0,07 \\
\hline Amebas & 0,15 & - & - \\
\hline Flagelados & 0,05 & $-0,05$ & $-0,04$ \\
\hline * $p<0,05$. & & &
\end{tabular}

Tabela 6 - Coeficientes de correlação entre os grupos biológicos do TA-2 e os parâmetros de processo (SS, SSD e OD)

\begin{tabular}{lccc} 
& \multicolumn{3}{c}{ Parâmetros } \\
\cline { 2 - 4 } Grupos & SS & SSD & OD \\
\hline Ciliados livre natantes & $-0,23^{\star}$ & 0,20 & $0,30^{\star}$ \\
\hline Ciliados de vida livre & $0,26^{\star}$ & 0,19 & 0,13 \\
\hline Ciliados pedunculados & $-0,18$ & 0,08 & $0,26^{\star}$ \\
\hline Amebas & 0,07 & $0,37^{*}$ & $-0,14$ \\
\hline Flagelados & $-0,34^{\star}$ & $-0,21$ & 0,05 \\
\hline
\end{tabular}

${ }^{*} p<0,05$.

Tabela 7 - Coeficientes de correlação entre os grupos biológicos do TA-2 e os parâmetros do ETF-1

\begin{tabular}{lcc} 
Grupos & SS & DQO \\
\hline Ciliados livre natantes & $-0,16$ & $-0,33^{*}$ \\
\hline Ciliados de vida livre & $-0,25^{*}$ & $-0,26^{*}$ \\
\hline Ciliados pedunculados & $-0,12$ & $-0,27^{*}$ \\
\hline Amebas & $-0,01$ & $-0,06$ \\
\hline Flagelados & $-0,21$ & $-0,18$ \\
\hline * $_{p}<0,05$. & &
\end{tabular}

a correlação existente fosse negativa, ou seja, quando do aumento da toxicidade do EI, a ocorrência dos grupos biológicos seria reduzida. A toxicidade do EI vem sendo determinada pelo sistema-teste Microtox (ARAÚJO et al, 2005), através da bactéria marinha Vibrio fischeri, de características e sensibilidade diferentes das dos organismos que compõem o lodo, o que deve explicar as correlações não significativas com a abundância dos organismos e a toxicidade do EI. Toda a complexidade em termos de diversidade biológica e diferenças fisiológicas dos organismos em questão devem ser levadas em conta (CHAPMAN, 2000; DALZELL et al, 2002).

A concentração de SS demonstrou exercer influência no TA-2, especialmente sobre os CLN, CVL e flagelados (Tabela 6). Houve correlação negativa entre SS e os CLN e flagelados. Por outro lado, com os CVL a correlação foi positiva. Usualmente, a quantidade de SS no licor misto é usada para estimar a quantidade de micro-organismo presente no lodo (YOKOI, 1999), especialmente quando nenhuma informação sobre o número destes é fornecida (ORUPÕLD; MASTRIN; TOOMAS, 2001). Os CVL são organismos considerados predadores de flocos estando associados a tais estruturas; desse modo, a correlação positiva encontrada com esse grupo pode ser assim explicada. No entanto, para os CLN e flagelados, a relação inversa com o teor de SS pode ter ocorrido pela não aderência desses organismos aos flocos, estando dispersos no licor misto. As amebas apresentaram correlação positiva com os teores de SSD (Tabela 6), o que pode ter ocorrido devido ao modo de vida dos organismos, especialmente pelo tipo de locomoção, que se dá por pseudópodos, requerendo substrato apropriado. Cutolo e Rocha (2000) verificaram correlação semelhante entre as amebas e o índice volumétrico do lodo (IVL), constituído pela relação entre sólidos sedimentáveis e sólidos suspensos. É importante destacar que apenas o aumento no teor de SSD do licor misto não levaria ao aumento na densidade de amebas no TA, pois outros fatores tais como toxicidade, amônia, DBO e DQO devem ser considerados. Os valores de OD apresentaram correlação positiva com os CLN e CP, indicando que, em boas condições de depuração, é provável o aparecimento dos organismos desses grupos (Tabela 6). As correlações positivas com OD são coerentes, pois o aumento de oxigênio, não excessivo, implica em maior eficiência (MENDONÇA, 2002). Os organismos do TA-3 e TA-4 não se correlacionaram significativamente com os parâmetros de processo avaliados, à exceção dos CLN que apresentaram correlação com o OD $(0,28)$ (dados não mostrados)

A presença dos ciliados (CLN, CP e CVL) demonstrou ser indicativo da qualidade do ETF-1 quanto à carga de DQO (Tabela 7). Maior frequência desses grupos indicou menor carga de DQO no ETF-1 e, consequentemente, melhor qualidade do efluente. Esses resultados estão em conformidade com os obtidos por Bento et al (2005), quando os ciliados foram associados à elevada remoção de DQO. Os ciliados têm grande importância nos processos de purificação de efluentes, pois removem a matéria orgânica dissolvida, como bactérias formadoras de flocos e partículas em suspensão, e clarificam o efluente por filtração (SALVADÓ; GRACIA; AMIGÓ, 1995; MADONI et al, 1996; RATSAK; MAARSEN; KOOIJMAN, 1996). Os flagelados não apresentaram correlação significativa com a carga de DQO do ETF-1. Esse grupo apresentou o mesmo comportamento registrado por Cutolo e Rocha (2000) quando observaram que os flagelados estão ligados ao processo de recuperação do sistema de tratamento após um choque de carga. Madoni (1994) mostrou que os flagelados têm influência negativa sobre o índice de qualidade do lodo, indicando má depuração ou, ainda, transição biológica (BENTO et al, 2005). Houve correlação negativa entre os 
CVL e a concentração de SS no ETF-1 (Tabela 7), o que corrobora com os dados obtidos com a DQO, quando os CVL demonstraram serem bons indicadores da qualidade do tratamento, uma vez que o elevado teor de SS no efluente tratado implica em menor eficiência do sistema no tratamento. Curds (1982) também observou um aumento de ciliados, seguido de redução no teor de SS, tendo explicado tal relação a partir da alimentação dos ciliados sobre as bactérias, que compreendem a maior parcela da biomassa do lodo ativado e dos sólidos liberados nos efluentes (DAVIES, 2005). O grupo dos CP demonstrou ser um importante indicador da qualidade do ETF-2 quanto à carga de DQO e SS. No TA-3, os flagelados e CLN também se correlacionaram negativamente com a carga de DQO de ETF-2 (Tabela 8).

A eficiência da ETE, durante este estudo, quanto à remoção de DQO, DBO e toxicidade foi de 94,5 $\pm 4,5 \%, 86,0 \pm 9,0 \%$ e $82,7 \pm$ $12,2 \%$, respectivamente, indicando elevado potencial de depuração dos efluentes. Tal cálculo foi feito apenas para a amostra composta do ETF, resultante do ETF-1 e ETF-2. Assim, pelo melhor desempenho do TA-2, e melhor qualidade do ETF-1, a eficiência desse TA deve ter sido subestimada. Os valores médios e os respectivos desvios-padrão dos parâmetros avaliados no EI, nos TA e nos ETF-1 e ETF-2 estão sumarizados na Tabela 9.

Durante todo o período de análise, as concentrações de OD no TA-4 foram extremamente baixas, com média de 0,32 mg/L, enquanto nos TA-2 e TA-3 foram 1,63 e 2,39 mg/L, respectivamente (Tabela 9). Quanto à diversidade biológica, o TA-4 também foi o menos diverso, caracterizando-se como o de menor eficiência. Devido à semelhança desse TA em relação ao TA-2, com mistura do tipo carrossel, acredita-se que um fator importante que culminou em baixa eficiência do TA-4 foi o déficit de oxigênio, reduzindo sua capacidade de biodegradação. De acordo com Davies (2005), a concentração de oxigênio é um fator limitante para as bactérias do lodo ativado, sendo exigido um mínimo de 1,2 mg/L para as bactérias formadoras de flocos e 0,6 mg/L para as bactérias filamentosas dispersas. Para o autor, abaixo desses valores, a taxa de respiração das bactérias reduz acentuadamente e favorece a proliferação de bactérias do tipo filamentosas anaeróbias facultativas. Durante o período estudado, a proliferação excessiva de bactérias filamentosas foi mais frequente no TA-4, gerando um efluente com pobre sedimentabilidade e elevada turbidez. A eficiência do TA-3 deve ter sido subestimada devido à junção do seu efluente tratado com o do TA-4, formando o ETF-2, pois esse último tanque teve desempenho inferior aos demais TA.

De modo geral, os coeficientes de correlação encontrados foram relativamente baixos. Correlações entre os organismos do lodo e variáveis de controle operacional não necessariamente são elevadas, especialmente com parâmetros cuja variação é grande (SALVADÓ; GRACIA; AMIGÓ, 1995), tais como os resultados apresentados (Tabela 9). Esses mesmos autores concluíram que existe uma faixa ótima de variação (decréscimo de desvio-padrão), na qual as correlações serão mais expressivas. Quando as análises foram realizadas

Tabela 8 - Coeficientes de correlação entre os grupos biológicos dos TA-3 e TA-4 e os parâmetros do ETF-2

\begin{tabular}{lcccc}
\multirow{2}{*}{ Grupos } & \multicolumn{2}{c}{ SS } & \multicolumn{2}{c}{ DQO } \\
\cline { 2 - 5 } & TA-3 & TA-4 & TA-3 & TA-4 \\
Ciliados livre natantes & $-0,20$ & $-0,01$ & $-0,29^{\star}$ & $-0,02$ \\
\hline Ciliados pedunculados & $-0,30^{\star}$ & $-0,11$ & $-0,32^{\star}$ & $-0,02$ \\
\hline Flagelados & $-0,26$ & $-0,23^{\star}$ & $-0,32^{\star}$ & $-0,14$ \\
\hline
\end{tabular}

$* p<0,05$.

Tabela 9 - Valores médios seguidos dos respectivos desvios-padrão dos parâmetros físico-químicos e ecotoxicológicos do El, do licor misto dos TA e dos ETF-1 e ETF-2

\begin{tabular}{|c|c|c|c|c|c|c|}
\hline \multirow{2}{*}{ Parâmetros } & \multicolumn{6}{|c|}{ Amostras } \\
\hline & EI & TA-2 & TA-3 & TA-4 & ETF-1 & ETF-2 \\
\hline $\mathrm{DBO}\left(\mathrm{mg} \mathrm{O}_{2} / \mathrm{L}\right)$ & $734,62(264,43)$ & - & - & - & - & - \\
\hline $\mathrm{DQO}\left(\mathrm{mg} \mathrm{O}_{2} / \mathrm{L}\right)$ & $1.547,02(385,16)$ & - & - & - & $294,60(130,14)$ & $331,68(107,56)$ \\
\hline $\mathrm{PH}$ & $7,94(0,28)$ & - & - & - & - & - \\
\hline $\mathrm{N}-\mathrm{NH}_{3}(\mathrm{mg} / \mathrm{L})$ & $60,89(28,38)$ & - & - & - & - & - \\
\hline TCO (mg/L.min) & $43,70(32,96)$ & - & - & - & - & - \\
\hline COT (mg/L) & $914,72(353,50)$ & - & - & - & - & - \\
\hline Toxicidade $\left(\mathrm{CE}_{50}\right)$ & $9,38(3,44)$ & - & - & - & - & - \\
\hline SS (mg/L) & - & $5.995,90(1138,92)$ & $7.134,28(1.797,67)$ & $7.268,42(2259,24)$ & $36,64(43,36)$ & $42,52(50,81)$ \\
\hline SSD (mL/L) & - & $394,54(176,29)$ & $366,06(167,61)$ & $392,23(198,43)$ & - & - \\
\hline OD (mg/L) & - & $1,62(0,70)$ & $2,39(1,35)$ & $0,32(0,46)$ & - & - \\
\hline
\end{tabular}


levando-se em consideração toda a variação das amostras, os coeficientes de correlação variaram entre 0,05 e 0,30, valores semelhantes aos encontrados neste estudo (SALVADÓ; GRACIA; AMIGÓ, 1995). A ausência de linearidade nas correlações, como documentada para o pH, pode ter ocorrido com alguns parâmetros contemplados neste estudo, resultando em coeficientes significativos, porém baixos.

\section{Conclusões}

Os organismos mais sensíveis a cargas elevadas de DBO e DQO foram os CVL, no TA-2, e CP nos TA-3. No TA-4 a abundância dos $\mathrm{CP}$ e flagelados foi a melhor referência. Quanto à elevada concentração de $\mathrm{N}-\mathrm{NH}_{3}$ no EIC, os CVL demonstraram maior sensibilidade. A presença dos ciliados (CLN, CP e CVL) foram os melhores indicadores da qualidade do tratamento dos efluentes do PIC, de modo que a presença dos organismos desse grupo indica boa depuração. Os CVL parecem indicar maior estabilidade e eficiência do lodo no tratamento do efluente.

O TA-2 demonstrou ser mais eficiente no tratamento de efluentes, devido à maior diversidade dos tipos morfológicos identificados. O TA-3 não demonstrou ser tão diverso quanto o TA-2, mas apresentou maior diversidade que o TA-4. Levando-se em conta que não há diferença no EI que alimenta os tanques, a diferença no sistema de mistura do TA-3 (mistura completa) pode ser o ponto crucial para que, mesmo com elevada concentração de OD, a eficiência seja menor que a do TA-2. O sistema do tipo carrossel parece ser mais adequado para essa ETE por proporcionar melhor mistura do efluente e maior contato com a biota do tanque. Para o TA-4, o maior problema quanto à eficiência na depuração do efluente deve estar relacionado ao déficit de OD no lodo.

Relações entre a comunidade presente no lodo e as características dos efluentes, sejam de entrada ou saída, e mesmo das condições operacionais impostas ao sistema, podem ser obtidas com grande representatividade da ETE. No entanto, essas relações precisam ser analisadas em conjunto com dados físicos, químicos e toxicológicos, especialmente quando se trata de efluentes complexos e com grande variação como os provenientes do PIC. As avaliações microscópicas do lodo mostraram-se adequadas para caracterização do funcionamento da ETE da Cetrel. Os dados aqui obtidos devem ser adotados seguramente como parâmetro de controle para a citada estação de tratamento, porém para as demais estações que operam com lodo ativado, devido às diferenças intrínsecas a cada sistema quanto à operação e estrutura da comunidade, devem ser vistos com cautela.

\section{Agradecimentos}

Os autores agradecem à Cetrel Proteção Ambiental pelo suporte, e aos pesquisadores C.B.A. Chastinet, F.F. Barbosa e P. Tadeu pelas contribuições ao trabalho. Agradecemos também aos valiosos comentários dos revisores deste trabalho.

\section{Referências}

AMMAN, R.; GLÖCKNER, F.O.; NEEF, A. Modern methods in subsurface microbiology: in situ identification of microorganisms with nucleic acid probes. FEMS Microbiology Reviews, v. 20, n. 3-4, p. 191-200, 1997.

APHA/AWWA/WEF. Standard Methods for the Examination of Water and Wastewater. 21. ed. Baltimore/Maryland: Port City Press/American Public Health Association, 2005.

ARAÚJO, C.V.M. et al. The use of Microtox to assess toxicity removal of industrial effluents from the industrial district of Camaçari (BA, Brazil) Chemosphere, v. 58, n. 9, p. 1277-1281, 2005.

BEAL, L.L. et al. Otimização de uma estação de tratamento de efluentes de uma indústria de embalagens de papel. Revista Engenharia Sanitária e Ambiental, v. 11, n. 3, p. 283-289, 2006.

BENTO, A.P. et al. Caracterização da microfauna em estação de tratamento de esgotos do tipo lodos ativados: um instrumento de avaliação e controle do processo. Revista Engenharia Sanitária e Ambiental, v. 10, n. 4, p. 329-338, 2005.
CETREL. Relatório da Gestão Prêmio Nacional de Qualidade (PNQ). Camaçari: Cetrel S.A, 2004.

CHAPMAN, P.M. Whole effluent toxicity testing: usefulness, level of protection, and risk assessment. Environmental Toxicology and Chemistry, v. 19, n. 1, p. 3-13, 2000.

COSTA, F.C. et al. Tratamento do efluente de uma indústria química pelo processo de lodos ativados convencional e combinado com carvão ativado. Revista Engenharia Sanitária e Ambiental, v. 8, n. 4, p. 274-284, 2003.

CURDS, C.R. The ecology and role of protozoa in aerobic sewage treatment processes. Annual Review of Microbiology, v. 36, p. 27-28, 1982.

CUTOLO, S.A.; ROCHA, A.A. Correlação entre a microfauna e as condições operacionais de um processo de lodos ativados. In: XXVII CONGRESSO INTERAMERICANO DE ENGENHARIA SANITÁRIA E AMBIENTAL. Porto Alegre, RS, Anais... Rio de Janeiro; ABES, 2000. 
DA MOTTA, M. et al. Estudo do funcionamento de estações de tratamento de esgotos por análise de imagem: validações e estudo de caso. Revista Engenharia Sanitária e Ambiental, v. 8, n. 3, p. 170-181, 2003.

DALZELL, D.J.B. et al. A comparison of five rapid direct toxicity assessment methods to determine toxicity of pollutants to activated sludge. Chemosphere, v. 47, n. 5, p. 535-545, 2002.

DAVIES, P.S. The biological basis of wastewater treatment. Glasgow, UK: Strathkelvin Instrument Ltd, 2005.

FERNANDES, J.G.S. et al. Utilização da respirometria no controle operacional de sistemas aeróbios de tratamento de águas residuárias: a experiência da Cetrel. Revista Engenharia Sanitária e Ambiental, v. 6, n. 3, p. 131-137, 2001.

FERNÁNDEZ, A. et al. Assessment of toxicity of river water and effluents by the bioluminescence assay using Photobacterium phosphoreum. Water Research, v. 29, n. 5, p. 1281-1286, 1995.

FORNEY, L.J. et al. Structure of microbial communities in activated sludge: potential implications for assessing the biodegradability of chemicals. Ecotoxicology and Environmental Safety, v. 49, n. 1, p. 4053,2001

FREIRE, R.S. et al. Novas tendências para o tratamento de resíduos industriais contendo espécies organocloradas. Química Nova, v. 23, n. 4, p. 504-511, 2000.

JENKINS, D.; RICHARD, M.G.; DAIGGER, G. T. Manual on the causes and control of activated sludge bulking, foaming, and other solids separation problems. 3. ed. Boca Raton: Lewis Publishers, 2003.

KUDO, R.R. Handbook of protozoology. Illinois: Charles C. Thomas Publisher, 1931.

LAMBOLEZ, L. et al. The environmental risks of industrial waste disposal: an experimental approach including acute and chronic toxicity studies. Ecotoxicology and Environmental Safety, v. 28, n. 3, p. 317-328, 1994

MADONI, P. A sludge biotic index (SBI) for the evaluation of the biological performance of activated sludge plants based on the microfauna analysis. Water Research, v. 28, n. 1, p. 67-75, 1994.

MADONI, P.; DAVOLI, D.; CHIERICI, E. Comparative analysis of the activated sludge microfauna in several sewage treatment works. Water Research, v. 27, n. 9, p. 1485-1491, 1993.

MADONI, P. et al. Toxic effect of heavy metals on the activated sludge protozoan community. Water Research, v. 30, n. 1, p. 135-141, 1996.

MENDONÇA, L.C. Microbiologia e Cinética de sistema de lodos ativados como pós-tratamento de efluente de reator anaeróbio de leito expandido. 219 p. Tese (Doutorado em Engenharia Civil) - Escola de Engenharia de São Carlos, Universidade de São Paulo, 2002.

MICROBICS CORPORATION. Microtox test manual. Carlsbad: California, USA, 1998
NEEDHAM, J.G.; NEEDHAM, P.R. Guia para el estudio de los seres vivos de las aguas dulces. Barcelona: Reverte, 1978.

OLIVEIRA, C.A. et al. Utilisation of respirometry to assess organic matter reduction of effluents from the industrial district of Camaçari (Ba, Brazil). Brazilian Archives of Biology and Technology, v. 50, n. 2, p. 311-319, 2007.

ORUPÕLD, K.; MAŠIRIN, A.; TOOMAS, T. Estimation of biodegradation parameters of phenolic compounds on activated sludge by respirometry. Chemosphere, v. 44, n. 5, p. 1273-1280, 2001.

PATOINE, A. et al. Toxicity reduction and removal of dehydroabietic and abietic acids in a continuous anaerobic reactor. Water Research, v. 31, n. 4, p.825-831, 1997

POOLE, J.E.P. A study of the relationship between the mixed liquor fauna and plant performance for a variety of activated sludge sewage treatment works. Water Research, v. 18, n. 3, p. 281-287, 1984.

RATSAK, C.H.; MAARSEN, K.A.; KOOIJMAN, A.L.M. Effects of protozoa on carbon mineralization in activated sludge. Water Research, v. 30, n. 1, p. 1-12, 1996

SALVADÓ, H.; GRACIA, M.P. Determination of organic loading rate of activated sludge plants based on protozoan analysis. Water Research, v. 27, n. 5, p. 891-895, 1993.

SALVADÓ, H.; GRACIA, M.P.; AMIGÓ, J.M. Capability of ciliated protozoa as indicators of effluent quality in activated sludge plants. Water Research, v. 29, n. 4, p. 1041-1050, 1995.

SANTOS, E.M.A. et al. Influência do tempo de detenção hidráulica em um sistema UASB seguido de um reator biológico com fungos para tratar efluentes de indústria de castanha de caju. Revista Engenharia Sanitária e Ambiental, v. 11, n. 1, p. 39-45, 2006.

UBAY ÇOKGÖR, E. et al. Respirometric analysis of activated sludge behaviour-I: assessment of the readily biodegradable substrate. Water Research, v. 32, n. 2, p. 461-475, 1998.

VAZOLLÉR, R.F. Microbiologia de processos biológicos de tratamento. In: IV CURSO DE TRATAMENTO BIOLÓGICO DE RESÍDUOS, Florianópolis, 1999.

VAZOLLÉR, R.F. et al. Microbiologia de lodos ativados. São Paulo: Cetesb, 1989.

VON SPERLING, M. Princípio do tratamento biológico de águas residuárias: lodos ativados. Minas Gerais: UFMG, 1997.

WESTPHAL, A. Zoología especial: protozoos. Barcelona: Omega, 1977.

YOKOI, H. et al. Growth inhibition of activated sludge by humus. Biotechnology Techniques, v. 13, n. 4, p. 263-266, 1999.

ZAR, J.H. Biostatistical analysis. 3. ed. USA: Ed. Prentice-Hall/Upper Saddle River, 1996. 